\title{
Método de Posicionamento Relativo por Satélite GPS com Correção do Efeito do Multicaminho em Estações de Referência: Formulação Matemática, Resultados e Análises ${ }^{1}$
}

W.G.C. POLEZEL 2 E.M. de SOUZA ${ }^{3}$, J.F.G. MONICO ${ }^{4}$ FCT, UNESP, 19060900 Presidente Prudente, SP, Brasil.

Resumo. O efeito do multicaminho afeta diretamente as observáveis do GPS, degradando o posicionamento. Quando se realiza o posicionamento pelo método relativo e a estação de referência GPS está afetada pelo multicaminho, os erros são propagados para a estação a ser determinada. Logo, apresenta-se algumas características mais relevantes desse efeito, bem como uma metodologia para a detecção do mesmo diretamente nas observações de fase e pseudodistância em estações de referência.

\section{Introdução}

Uma tecnologia que já faz parte da vida, não só da comunidade científica, mas também de usuários em geral, é o GPS (Global Positioning System). Devido a precisão que o GPS possibilita no posicionamento, ele tem sido utilizado para diversos fins operacionais, desde uma simples navegação por aventureiros, até na realização de posicionamento de ordem milimétrica.

Entretanto, existem alguns erros que degradam a precisão do posicionamento. Dentre os erros envolvidos, pode-se citar os erros devido ao relógio do satélite, órbitas, efeitos atmosféricos, erro do relógio do receptor e efeito do multicaminho. Tais erros deterioram a qualidade das observáveis GPS (código e fase da onda portadora) e precisam ser tratados adequadamente, para que seja possível realizar posicionamento de ordem milimétrica. Esse tratamento se faz necessário devido à impossibilidade do modelo matemático representar a realidade física com total fidelidade [1]. A maioria dos erros envolvidos pode ser minimizada no posicionamento relativo através de combinações lineares na modelagem funcional. Entretanto, o efeito do multicaminho, o qual ocorre devido às reflexões do sinal em superfícies (edifícios, lagos, veículos) antes de chegar ao receptor, não pode ser eliminado pois o multicaminho é um efeito altamente dependente da localização de cada receptor.

\footnotetext{
${ }^{1}$ Trabalho financiado pela FAPESP - Processos 2006/05410-9 e 03/12770-3,

${ }^{2}$ wesleypolezel@hotmail.com, Programa de Pós Graduação em Ciências Cartográficas,

3 eniuce@yahoo.com.br, Programa de Pós Graduação em Ciências Cartográficas,

${ }^{4}$ galera@fct.unesp.br, Departamento de Cartografia.
} 
Neste sentido, objetiva-se neste artigo apresentar as formulações matemáticas envolvidas no posicionamento relativo, bem como o método desenvolvido para correção do efeito do multicaminho.

\section{Posicionamento Relativo}

No posicionamento relativo, a posição de um ponto é determinada com relação à de outro(s), cujas coordenadas são conhecidas [3]. Estes pontos com coordenadas conhecidas são chamados de estações de referência ou estações base.

Para realizar o posicionamento relativo, o usuário deve utilizar dados de dois ou mais receptores que rastreiem, simultaneamente, os mesmos satélites. Como estação de referência o usuário pode utilizar os dados da RBMC (Rede Brasileira de Monitoramento Contínuo). Se a estação de referência possui erros, tais como o multicaminho, estes são propagados para as coordenadas do usuário. Logo, é importante que esses efeitos sejam corrigidos em estações de referência para que as coordenadas obtidas pelos usuários a partir deste método de posicionamento sejam mais acuradas.

O posicionamento relativo pode ser realizado utilizando as seguintes observáveis: pseudodistância (PD) e fase da onda portadora.

A PD é determinada pelo tempo de propagação do sinal, contado desde a sua geração no satélite até o receptor multiplicado pela velocidade da luz no vácuo e outros fatores que interferem no sinal (efeitos atmosféricos e multicaminho). A PD pode ser modelada pela seguinte equação [3]:

$$
P D_{r}^{s}=\rho_{r}^{s}+d \rho_{r}^{s}+c\left[d t_{r}-d t^{s}\right]+I_{r}^{s}+T_{r}^{s}+d m_{P D r}^{s}+\varepsilon_{P D_{r}^{s}},
$$

onde

- $c$ é a velocidade da luz $(\mathrm{m} / \mathrm{s})$;

- $\rho_{r}^{s}$ é a distância geométrica entre o satélite s no instante de transmissão do sinal e o receptor $\mathrm{r}$ no instante de recepção $(\mathrm{m})$;

- $d \rho_{r}^{s}$ é o erro em $\rho$ geralmente devido ao erro da órbita do satélite $(\mathrm{m})$;

- $d t^{s}$ e $d t_{r}$ são os erros do relógio do satélite em relação ao tempo GPS no instante $\mathrm{t}_{s}$ e $\mathrm{t}_{r}(\mathrm{~s})$, ou seja, o tempo de recepção e de transmissão do sinal;

- $I_{r}^{s}$ e $T_{r}^{s}$ são os efeitos ionosférico e troposférico $(\mathrm{m})$;

- $d m_{P D r}^{s}$ é o erro de multicaminho na PD (m);

- $\varepsilon_{P D_{r}^{s}}$ é o erro da PD devido aos efeitos não modelados (m).

Para a medida da fase de batimento da onda portadora, a qual é determinada a partir da diferença entre a fase gerada pelo satélite $\left(\varphi^{s}\right)$, no instante de transmissão do sinal, e sua réplica gerada pelo receptor $\left(\varphi_{r}\right)$, no instante de recepção do sinal. Apenas uma medida fracionária é obtida, na primeira época, restando um número inteiro de ciclos no instante inicial do rastreio, denominado de ambigüidade $\left(N_{r}^{s}\right)$ [3]. A equação da fase de batimento da onda portadora $\varphi_{r}^{s}$ é dada por

$$
\varphi_{r}^{s}=f\left(\frac{\rho_{r}^{s}+d \rho_{r}^{s}-I_{r}^{s}+T_{r}^{s}+d m_{\varphi r}^{s}}{c}\right)+f\left(d t_{r}-d t^{s}\right)+\left[\varphi^{s}\left(t_{0}\right)-\varphi_{r}\left(t_{0}\right)\right]+N_{r}^{s}+\varepsilon_{\varphi r}^{s},
$$

onde $f$ é a freqüência nominal da fase (Hertz), $\varphi^{s}\left(t_{0}\right)$ é a fase inicial do satélite e $\varphi_{r}\left(t_{0}\right)$ é a fase recebida no receptor corresponde à época $t_{0}$ (ciclos), $d m_{\varphi_{r}}^{s}$ é o erro 
de multicaminho da fase $(\mathrm{m})$ e $\varepsilon_{\varphi_{r}}^{s}$ é o erro da fase da onda portadora devido a efeitos não modelados (ciclos).

\subsection{Combinações lineares das observáveis GPS}

O método de posicionamento relativo baseia-se em realizar combinações lineares das observáveis de duas estações (que se deseja determinar e a estação base) e reduzir ao máximo os erros envolvidos [1]. Estas observáveis secundárias que vem das originais são usualmente denominadas simples e duplas diferenças ([2] e [3]).

\subsubsection{Simples diferença}

Considerando dois receptores e um satélite, pode formar uma simples diferença (SD) [3]. A idéia fundamental é que os receptores $\left(r_{1}\right.$ e $\left.r_{2}\right)$ estejam rastreando simultaneamente o mesmo satélite (Figura 1).

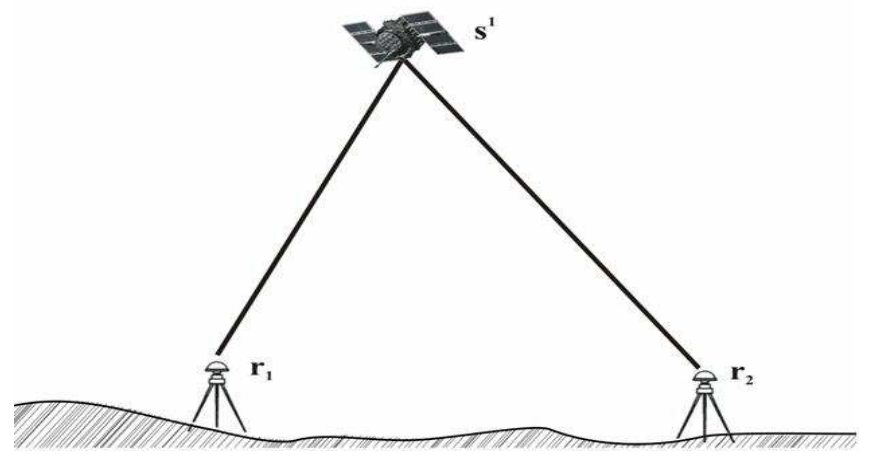

Figura 1: Simples diferença formada entre dois receptores

Quando uma combinação linear é realizada entre as observáveis das PD de duas estações tem-se a SD da PD A equação da SD da PD é obtida a partir de [3]:

$$
\Delta P D_{1,2}^{1}=\Delta \rho_{1,2}^{1}+c\left(d t_{r 1}-d t_{r 2}\right)+\Delta d m_{P D r 1,2}^{1}+v_{\Delta P D},
$$

onde $\Delta$ representa a SD entre os receptores de forma que $\Delta \rho_{1,2}^{1}=\rho_{1}^{1}-\rho_{2}^{1}$ e $\Delta d m_{P D r 1,2}^{1}=d m_{P D r 1}^{1}-d m_{P D r 2}^{1}$, e $v_{\Delta_{P D}}$ resíduo da $\mathrm{SD}$ da $\mathrm{PD}(\mathrm{m})$.

Da mesma forma, a SD da fase da onda portadora é expressa por [3]

$\Delta \varphi_{1,2}^{1}=\frac{f}{c}\left(\Delta \varphi_{1,2}^{1}+\Delta d \varphi_{1,2}^{1}+\Delta d m_{\varphi r_{1,2}}^{1}\right)+f\left(d t_{r_{1}}-d t_{r_{2}}\right)+\Delta \varphi_{1,2}\left(t_{0}\right)+\Delta N_{1,2}^{1}+v_{\Delta \varphi}$ onde $\Delta \varphi_{1,2}^{1}=\varphi_{1}^{1}-\varphi_{2}^{1}, \Delta d m_{\varphi r 1,2}^{1}=d m_{\varphi r 1}^{1}-d m_{\varphi r 2}^{1}, \Delta N_{1,2}^{1}=N_{1}^{1}-N_{2}^{1}, \Delta \varphi_{1,2}\left(t_{0}\right)=$ $\varphi_{1}\left(t_{0}\right)-\varphi_{2}\left(t_{0}\right)$ e $v_{\Delta \varphi}$ é resíduo da $\mathrm{SD}$ da fase (ciclos).

$\mathrm{Na}$ SD vários tipos de erros são praticamente eliminados, por exemplo, o erro do relógio do satélite e os erros devido à órbita do satélite. Para linhas de base curtas, os efeitos da ionosfera e troposfera são similares, sendo, portanto, praticamente eliminados. Os erros não modelados ou não totalmente eliminados são assumidos como de natureza aleatória, fazendo parte do resíduo da observação em questão. 
Entretanto, o multicaminho não é reduzido, pois depende da geometria entre o receptor, satélite e refletor, ou seja, das condições de reflexão do sinal na região onde o receptor está localizado [3].

\subsubsection{Dupla diferença}

A dupla diferença (DD) é a combinação linear dada pela diferença entre duas SD. Envolve, dois receptores e dois satélites [3] onde um destes satélites é considerado como base e utilizado nas demais combinações. Na Figura 2 pode-se observar a DD.

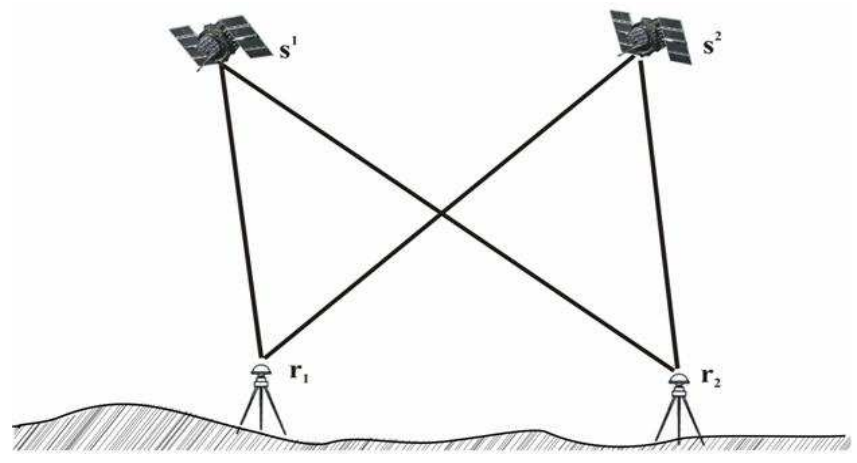

Figura 2: Dupla diferença

A equação da DD para a PD é expressa por [3]

$\nabla \Delta P D_{1,2}^{1,2}=\nabla \Delta \rho_{1,2}^{1,2}+\nabla \Delta d m_{P D 1,2}^{1,2}+v_{\nabla \Delta P D}$,

onde $\nabla$ representa a diferença entre os satélites, $\Delta \nabla \rho_{1,2}^{1,2}=\Delta \rho_{1,2}^{1}-\Delta \rho_{1,2}^{2}$, $\Delta \nabla d m_{P D r 1,2}^{1,2}=\Delta d m_{P D r 1,2}^{1}-\Delta d m_{P D r 1,2}^{2}$ e $v_{\nabla \Delta P D}$ é resíduo da DD da PD (m).

De forma similar, a equação para a fase é expressa da forma [3]: $\nabla \Delta \varphi_{1,2}^{1,2}=$ $\frac{f}{c}\left(\nabla \Delta \rho_{1,2}^{1,2}+\nabla \Delta d m_{\varphi r 1,2}^{1,2}\right)+\nabla \Delta N_{1,2}^{1,2}+v_{\nabla \Delta \Delta \varphi}$, onde $\Delta \nabla N_{1,2}^{1,2}=\Delta N_{1,2}^{1}-\Delta N_{1,2}^{2}$, $\Delta \nabla d m_{P D r 1,2}^{1,2}=\Delta d m_{P D r 1,2}^{1}-\Delta d m_{P D r 1,2}^{2}$ e $v_{\nabla \Delta \varphi}$ é o resíduo da DD da fase (ciclos).

A característica mais importante das DD é que nas combinações entre duas SD os erros dos relógios dos receptores são eliminados na equação da PD. Porém, da mesma forma que para a SD, o multicaminho não é eliminado na DD.

A equação de DD é normalmente a combinação linear preferida no modelo funcional para o processamento de dados GPS envolvendo a fase da onda portadora. A DD utilizando a fase proporciona melhor relação entre o ruído resultante e a eliminação de erros sistemáticos envolvidos nas observáveis originais [3].

A partir destes modelos matemáticos a determinação dos parâmetros desconhecidos (as coordenadas) é geralmente baseada no Método dos Mínimos Quadrados (MMQ). O ajustamento de observações pelo MMQ pode ser efetuado utilizando o método das equações de observação (paramétrico) ([1] e [2])

$$
L=L_{0}-L_{b}, \quad N=A^{T} P A, \quad U=A^{T} P L, \quad X=-N^{-1} U, \quad V=A X-L_{b},
$$


onde $L_{0}=F\left(X_{0}\right)$ é o vetor $(2 n \times 1)$ dos parâmetros aproximados, $L_{b}$ é o vetor $(2 n \times 1)$ das observações (PD e fase), $P$ é a matriz $(2 n \times 2 n)$ peso, $V$ é o vetor dos resíduos $(2 n \times 1)$ e $A$ é a matriz $(2 n \times u)$ design ou jacobiana do ajustamento é dada por uma aproximação linear das equações de observações considerando o primeiro termo da série de taylor em série matricial [1].

É importante observar que o efeito do multicaminho é o erro predominante na equação (2.1) dos resíduos $V$ das DD, pois esse foi o único efeito não reduzido pela combinação linear de DD adotada no modelo funcional.

\section{Mitigação do Multicaminho}

Foi desenvolvido um método para determinar e corrigir o efeito do multicaminho em estações de referência. Neste método, é necessário instalar pelo menos dois receptores em locais próximos da estação de referência em que se deseja estimar os valores de multicaminho. Tais locais devem ser escolhidos de forma que não haja superfícies refletoras significativas nas proximidades.

Inicialmente realiza-se o método de posicionamento relativo (seção 2) utilizando como estações base os receptores "livres" do multicaminho que são instalados nas proximidades da estação de referência. Após este procedimento, os resíduos das DD podem ser utilizados para determinar o erro do multicaminho.

De acordo com as considerações anteriores, a equação da DD dos resíduos (equação 2.1) para a PD ou fase pode ser expressa

$$
\nabla \Delta V_{a, b}^{S 1, S 2}=\Delta V_{a, b}^{S 1}-\Delta V_{a, b}^{S 2},
$$

onde o índice $a$ representa o receptor base e o índice $b$ a estação contínua, enquanto $S 1$ e $S 2$ representam os satélites envolvidos nas DD.

Considerando o erro do multicaminho como dominante nos resíduos, tem-se

$$
\nabla \Delta V_{a, b}^{S 1, S 2}=M_{a}^{S 1}-M_{b}^{S 1}+M_{b}^{S 2}-M_{a}^{S 2}+v,
$$

onde $M_{i}^{j}$ é o erro de multicaminho na estação $i$ e em relação ao satélite $j$, e $v$ representa os demais erros contidos no resíduo.

Como a estação base é "livre" do multicaminho, as componentes dos resíduos relativas ao multicaminho nas medidas observadas podem ser consideradas nulas. Logo, a equação 2.1 se reduz a: $\nabla \Delta V_{a, b}^{S 1, S 2}=-M_{b}^{S 1}+M_{b}^{S 2}+v$.

Como resultado, tem-se um sistema de $n$ equações e $u$ incógnitas para cada observável (PD e fase) em um determinado instante $t$. Neste caso, $u$ é a quantidade de satélites e $n$ é igual a quantidade de resíduos de DD multiplicada pelo número de linhas de base. No caso em que se têm duas linhas de base, obtém-se $2 n$ resíduos de DD, ou seja, $2 n$ equações com $2 n-u$ graus de liberdade. Pode-se então realizar um ajustamento pelo método paramétrico utilizando os valores dos resíduos das DD como observações e determinar o erro de multicaminho nas medidas de PD e fase para cada satélite visível na estação de referência. Entretanto, a matriz $N$ (equação 2.1) obtida é singular, o que requer injunções adicionais. O sinal do satélite com maior ângulo de elevação (utilizado como satélite base) pode ser considerado como 
quase isento de efeito do multicaminho. De acordo com esta consideração, foram introduzidas injunções de $1 \mathrm{~mm}$ para o multicaminho da fase e $3 \mathrm{~cm}$ para a PD para os dados do satélite base, porém, com o peso pequeno para esse satélite $\left(10^{-7}\right)$.

$\mathrm{O}$ erro de multicaminho nas medidas de $\mathrm{PD}$ e fase deve ser determinado durante alguns dias consecutivos, pois se o ambiente onde a estação de referência está instalada permanece inalterado, esse efeito muda de acordo com o movimento do satélite, e conseqüentemente, se repete em dias consecutivos. Esta característica é chamada de repetibilidade e permite avaliar a eficiência do método proposto.

Para aplicar este método, desenvolveu-se um software chamado EMR (Estimador de Multicaminho em estação de Referência) em linguagem $\mathrm{C} / \mathrm{C}++$.

\section{Experimento}

Para aplicar e avaliar o método proposto para correção do multicaminho, foi utilizada a estação de referência PPTE, pertencente à RBMC. Esta estação fica localizada na UNESP em Presidente Prudente. Foram instalados dois receptores em dois pilares formando duas linhas de base: uma de 19m (P1-PPTE) e outra de 20,3m (P2-PPTE). Os três receptores envolvidos no experimento (PPTE, P1 e P2) foram do mesmo modelo. Devido à altura e localização dos pilares, pode-se desconsiderar o efeito do multicaminho provindo de reflexões em objetos no horizonte. O cenário do experimento é ilustrado na Figura 3.

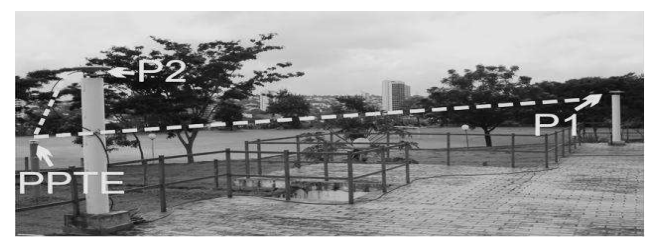

Figura 3: Local do experimento

Embora os dados tenham sido coletados continuamente entre os dias 15/12/2005 e 14/01/2006, o processamento dos dados foi realizado para apenas o período das $6: 34 \mathrm{~h}$ às $7: 49 \mathrm{~h}$. A coleta de dados foi realizada em diferentes dias para analisar a repetibilidade do efeito do multicaminho.

\subsection{Resultados e análises}

O PRN 18 (Pseudo-Random-Noise) foi escolhido como satélite base nas combinações lineares de DD, pois este satélite apresentava o maior ângulo de elevação $\left(65^{\circ}\right.$ a $\left.70^{\circ}\right)$. Satélites com maiores ângulos de elevação são, geralmente, menos propícios a causar reflexões. Os resíduos da DD envolvendo os PRN 18 e 21 foram utilizados para mostrar os resultados. O mesmo ocorreu nos demais PRN. Devido a grande quantidade de dados e para facilitar a visualização dos gráficos, os resultados serão mostrados apenas para dois dias. 


\subsubsection{Detecção do multicaminho e indicativos da validação do método}

Se o multicaminho é predominante nos resíduos do ajustamento, estes devem apresentar uma repetibilidade entre diferentes dias. Isso também deve ser verificado no multicaminho estimado a partir dos resíduos das duas linhas de base. Estas características representam indicativos de validade do método proposto.

Os resíduos da DD 18-21 são ilustrados na Figura 4 para os dias 4 e 5/01/2006 na linha de base P1-PPTE. Na Figura 4, embora os ruídos nos resíduos dificultem um pouco a visualização, observa-se o comportamento similar entre os dias, o qual caracteriza a predominância do multicaminho.

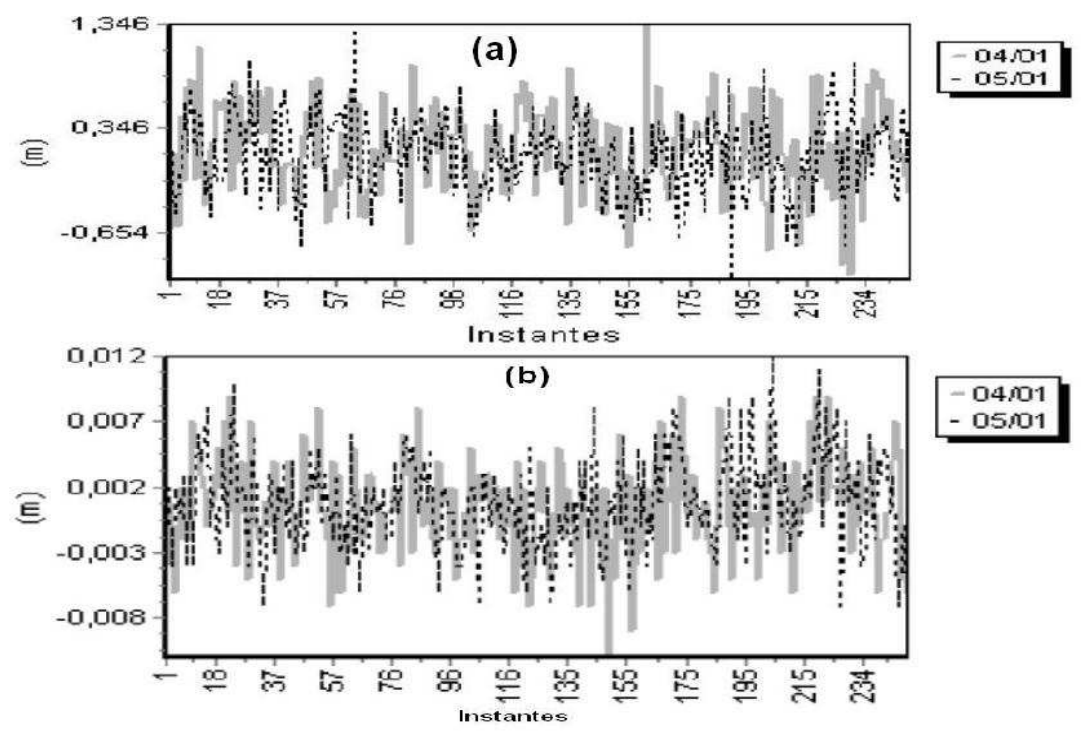

Figura 4: Resíduos da PD (a) e fase (b) na DD 18-21 para linha de base P1-PPTE

Analogamente, na Figura 5 são plotados os resíduos da DD 18-21 para P2PPTE. Observa-se que o multicaminho para P1-PPTE se repetiu de forma similar para P2-PPTE entre os diferentes dias.

Do escopo apresentado, pode-se concluir que há uma repetibilidade do multicaminho nas duas linhas de base nos resíduos das DD.Em seguida, os valores do multicaminho foram determinados a partir do software EMR. Na Figura 6 são apresentados os valores obtidos do multicaminho na estação de referência para o PRN 21 em relação às observáveis $\mathrm{PD}$ e fase. Na Figura 6 nota-se o comportamento similar do multicaminho entre os diferentes dias para o PRN 21, para a PD e fase. Logo, tem-se indicativos de eficiência da metodologia proposta devido à repetibilidade.

\subsubsection{Aplicação das correções do efeito do multicaminho}

Uma vez determinado o multicaminho para cada dia (de 1 a 5/01/2006), é necessário fazer uma média destes valores para obter uma única série temporal de correções. 

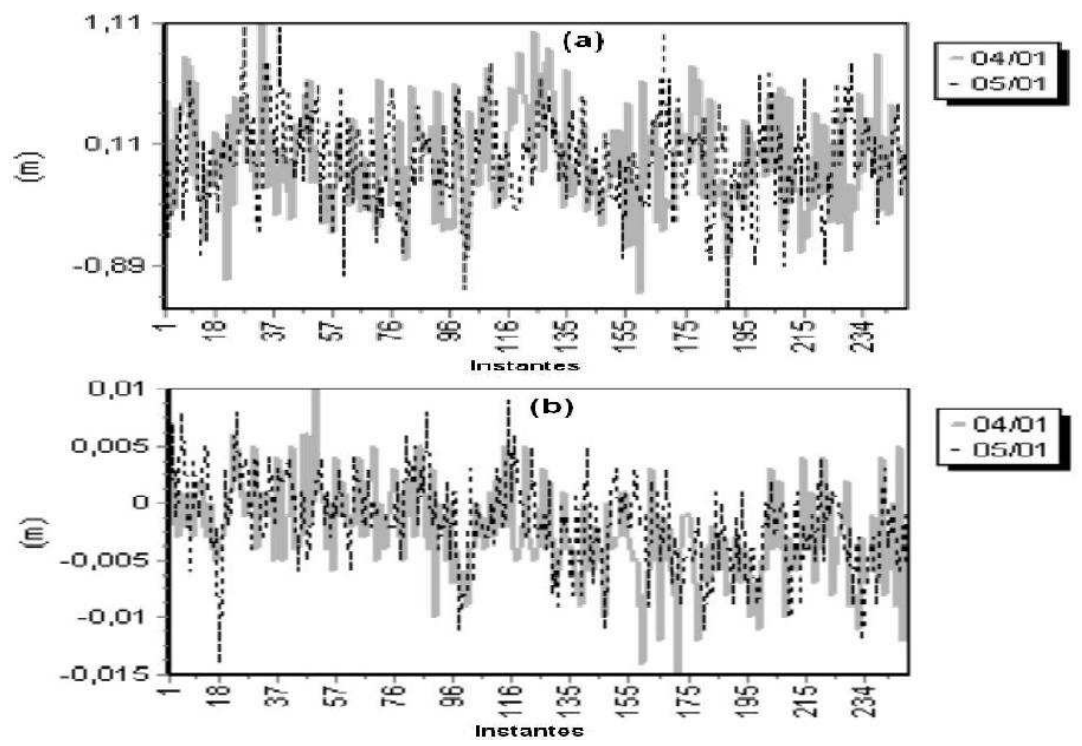

\section{$\begin{array}{r}-04 \pi 01 \\ -05 \pi 1 \\ \hline\end{array}$}

Figura 5: Resíduos da PD (a) e fase (b) da DD 18-21 para linha de base P2-PPTE

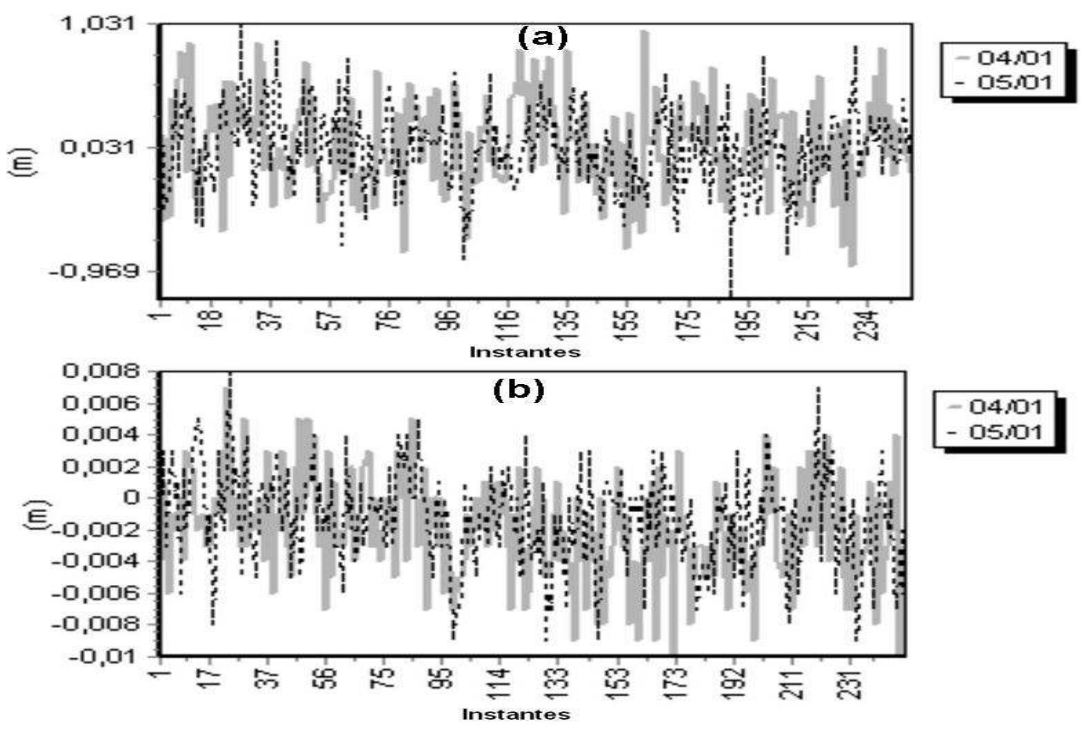

Figura 6: Erro do multicaminho da PD (a) e fase (b) no PRN 21 na estação PPTE

Esta série foi aplicada nos dias 27 a 31/12/2005 e 6 a 10/01/2006 (5 dias antes e 5 depois do período utilizado para determinar a série temporal das correções do multicaminho através da média).

Aplicando as correções do multicaminho nas observáveis de PD e fase da estação 
PPTE para cada instante, os dados dessa estação estão, teoricamente, livres do multicaminho. Ao realizar o posicionamento relativo utilizando os dados da estação base corrigidos do multicaminho, deve-se obter resultados melhores.

Com o intuito de analisar e comparar a qualidade das observações na DD para a PD e para a fase, adotando ou não a método proposto, utilizou-se o teste estatístico GOM (Global Overall Model)[6], o qual é sensível aos erros globais não modelados, como o caso do multicaminho. Este teste envolve as observações desde o instante $k_{0}$ até o instante $k: T_{G O M}^{K_{0}, K}=\frac{\sum_{i=K_{0}}^{K} v_{i}^{T} Q_{v_{i}}^{-1} v_{i}}{\sum_{i=l}^{k} m_{i}}$. O erro é detectado quando $T_{G O M}^{k}$ exceder o valor do teste Qui-Quadrado $\left(\chi^{2}\right)$, a um determinado nível de significância a partir da MVC dos resíduos $\left(Q_{v_{i}}^{-1}\right)$ por um grau de liberdade $m_{i}$.

A partir dos valores do GOM, observou-se que a qualidade das observações melhorou. Na Tabela 1 são apresentados os valores médios e desvios padrão do teste GOM, sem (GOMs) e com (GOMc) correção desse efeito.

Tabela 1: Valores médios e desvios padrão (DP) das estatística GOMs e GOMc.

\begin{tabular}{rccc}
\hline \multicolumn{2}{c}{ Linhas de base } & PPTE-P1 & PPTE-P2 \\
\hline \multirow{2}{*}{ GOMs } & Med. & 2,012 & 2,240 \\
& DP & 0,074 & 0,104 \\
\hline \multirow{2}{*}{ GOMc } & Med. & 1,173 & 1,340 \\
& DP & 0,534 & 0,567 \\
\hline Melhoria & Fator de melhoria & 1,7 & 1,7 \\
\hline
\end{tabular}

Na Tabela 1 pode-se observar que o teste GOM melhorou nas duas linhas de base indicando, em média, que os erros foram reduzidos por um fator de 1,7.

Analisou-se também as coordenadas estimadas, com e sem correção do multicaminho e foram comparadas com as coordenadas conhecidas.Foram calculadas as médias das resultantes horizontais e a componente vertical, sem e com a correção do multicaminho, para as estações P1 e P2 de 27 a 31/12/2005 e 6 a 10/01/2006.

Os valores das médias das discrepâncias das coordenadas para a estação P1, em geral, apresentaram poucas melhorias quando se eliminou o multicaminho nas observáveis. As resultantes, melhoraram na ordem de 0,18 mm.

Em relação à estação P2, todos os valores das diferenças entre as médias das coordenadas sem e com a correção do multicaminho melhoraram, atingindo até 2,4 $\mathrm{mm}$ de melhoria na discrepância das resultantes. Dessa forma, verificou-se que as coordenadas ficaram um pouco mais acuradas quando este efeito foi corrigido. Estas melhorias foram muito pequenas devido ao fato da estação de referência PPTE não estar em um local que tenha erros de multicaminho significativos.

\section{Conclusão}

A determinação e correção do erro de multicaminho em estações de referência podem trazer importantes contribuições aos que necessitam de posicionamento de alta 
precisão. Na detecção, os valores do multicaminho no GPS são estimados a partir dos resíduos das medidas de DD da PD e fase. Já na correção, os valores estimados para o multicaminho são corrigidos nas observações da estação de referência.Os resultados foram comparados sem e com a aplicação do método proposto. O método mostrou-se eficiente em razão da repetibilidade do multicaminho.

Em relação à qualidade das observações, o teste estatístico GOM indicou que os erros foram reduzidos com fator de 1,7. Isto demonstra que tal método melhorou a qualidade das observações na estação PPTE. Já nas coordenadas, a melhoria não foi significativa, em razão do multicaminho ser de ordem de grandeza muito pequena.

Abstract. The multipath effect affects the GPS observables, degrading the positioning. When the positioning is accomplished using relative method and the GPS reference station is affected by multipath effect, the errors are propagated to the station that is being determinated.Thus the aim of this paper is to present some of the most relevant features of multipath effect and a methodology to detect this effect directly in the carrier phase and pseudorange observables in reference stations.

\section{Referências}

[1] C. Gemael, "Introdução ao Ajustamento de Observação: Aplicação Geodésica", Ed. UFPR, Curitiba, 1994.

[2] A. Leica, "GPS Satellite Surveying", ed. 3, John Wiley \& Sons, Canada, 2004.

[3] J.F.G. Monico, "Posicionamento pelo NAVSTAR - GPS: Descrição, Fundamentos e Aplicações", UNESP, São Paulo, 2000.

[4] G. Seeber, "Satellite Geodesy: Foundations, Methods, and Applications", Walter de Gruyter, Berlin, New York, 2003.

[5] E.M. Souza, "Efeito de Multicaminho de Alta Frequência no Posicionamento Relativo GPS Estático: Detecção e atenuação utilizando wavelets", Dissertação (Mestrado em Ciências Cartográficas), UNESP/FCT, Presidente Prudente, 2004 .

[6] P.J.G. Teunissen, "Quality Control and GPS". In: P.J.G. Teunissen; A. Kleuberg, GPS for Geodesy, 2 ed., Springer Verlage, Berlin, 1998. 